\title{
Electrochemical fabrication of conducting polymer nanowires in an integrated microfluidic system
}

\author{
Jun Wang, ${ }^{a}$ Yuri L. Bunimovich, ${ }^{b}$ Guodong Sui, ${ }^{a}$ Stavros Savvas, ${ }^{a}$ Jinyi Wang, ${ }^{a}$ Yaoyao Guo, ${ }^{a}$ \\ James R. Heath* ${ }^{*}$ and Hsian-Rong Tseng*a
}

Received (in Cambridge, UK) 30th March 2006, Accepted 26th May 2006

First published as an Advance Article on the web 14th June 2006

DOI: $10.1039 / \mathrm{b} 604426 \mathrm{c}$

In this paper, we introduce a new approach for the in situ electrochemical fabrication of an individually addressable array of conducting polymer nanowires (CPNWs) positioned within an integrated microfluidic device and also demonstrate that such an integrated device can be used as a chemical sensor immediately after its construction.

The development of one-dimensional (1-D) nanomaterials for the ultrasensitive detection of biological species continues to receive widespread attention, with significant progress having been achieved in the use of silicon nanowires ${ }^{1}$ and carbon nanotubes ${ }^{2}$ for the detection of, among other things, proteins and DNA. Conducting polymer nanowires (CPNWs) are attractive alternatives to silicon nanowires and carbon nanotubes because of their tunable conductivity, flexibility, chemical diversity, and ease of processing. CPNWs can be prepared using a variety of protocols, such as chemical synthesis, ${ }^{3}$ templated electrochemical synthesis, ${ }^{4}$ and electro-spinning, ${ }^{5}$ and some chemical and biological sensors based on CPNWs have been reported. ${ }^{6}$

In general, the fabrication of 1-D nanomaterial-based electronic biosensors involves three distinct steps: (i) production of 1-D nanomaterials, (ii) merging 1-D nanomaterials into lithographically defined electrodes, and (iii) integration of electronic and microfluidic components. Great efforts are required during the post-production of 1-D nanomaterials incorporating those nanowires if they are to be used as functioning biosensors. There is a definite need for the development of much more convenient technologies. In this paper, we introduce a new approach that simplifies the three steps into one: the in situ electrochemical fabrication of an individually addressable array of CPNWs positioned within an integrated microfluidic device. We also demonstrate that such an integrated device can be used as a chemical sensor immediately after its construction.

Microfluidic technology ${ }^{7}$ is currently being used for diverse applications, including chemical synthesis, ${ }^{8}$ separation, ${ }^{9}$ and diagnosis. ${ }^{10}$ Multilayer PDMS-based microfluidic systems possessing integrated valves and pumps $^{7 d}$ have been developed to perform multiple functions within the same fluidic circuit. ${ }^{7}$ Recently, several groups have used microfluidics to fabricate nanomaterials such as silicon colloids ${ }^{11}$ and vesicles. ${ }^{12}$ Microfluidic

${ }^{a}$ Department of Molecular Medical Pharmacology and Crump Institute for Molecular Imaging. University of California, Los Angeles,

700 Westwood Plaza, Los Angeles, CA 90095, USA.

E-mail: hrtseng@mednet.ucla.edu; Fax: 1-310-206-8975;

Tel: 1-310-794-1977

${ }^{b}$ Division of Chemistry and Chemical Engineering, California Institute of Technology, Pasadena, CA 91125, USA.E-mail: heath@its.caltech.edu environments add value to biosensing tasks because they consume lower amounts of probe molecules and target analytes.

There are certain key advantages to preparing CPNWs within a microfluidic device using spatially localized, template-free electrochemical polymerization: (i) the monomeric precursor polymerizes directly on the electrode surface, producing high-quality ohmic contacts; (ii) addressability is inherent to this method because nanowires can be grown across individual electrode junctions; (iii) the introduction and delivery of small amounts of precursor monomers and analytes are highly controllable and enable the rapid exchange of nanoliter-level solutions on the same chip; (iv) the turbulence-free environment ${ }^{13}$ within a microchannel helps the formation of well-defined CPNWs during the electropolymerization process; and (v) once the nanowires are grown, the entire device is ready for use, without the necessity of any postfabrication processing.

Fig. 1a and b display images of the microfabricated and assembled integrated microfluidic device. The device comprises an array of Pt working microelectrodes (each pair separated by a $2 \mu \mathrm{m}$-wide gap) and a single platinum counter electrode, which are positioned within a microchannel of an overlaying two-layer PDMS microfluidic component. The reference $\mathrm{Ag} / \mathrm{AgCl}$ electrode is placed just downstream of the working electrodes. We fabricated $\uparrow$ this microfluidic chip by using a previously described procedure. $^{7 \mathrm{~b}-\mathrm{d}}$ The input channels can be used to deliver both the monomer precursor solutions for nanowire growth and the analyte solutions for nanowire sensing. Pressurized control lines are used to select from among the input solutions. The widths and heights of the channels are 100 and $16 \mu \mathrm{m}$, respectively. We used standard photolithography techniques to fabricate the Pt microelectrodes on a silicon wafer possessing a thermally grown oxide layer. Initially, the integrated microfluidic device was completely filled with deionized water. By controlling the isolation valves, ${ }^{7 \mathrm{~d}}$ a solution of pyrrole or aniline monomer (driven by a back pressure of 2 psi) can be specifically introduced to the electrode junctions. After the monomer solution was delivered to the electrode junctions, one electrode on either side of the junction served as the working electrode for the electropolymerizations of the corresponding CPNWs (Fig. 1c).

We employed the galvanostatic current step method ${ }^{6 \mathrm{~b}, 6 \mathrm{c}, 14}$ for the fabrication of the CPNWs. We applied an initial current of $0.4-0.8 \mathrm{nA}$ to the electrode for $50-300 \mathrm{~s}$, reduced the current to $0.2-0.4 \mathrm{nA}$ for $500-1000 \mathrm{~s}$, and then reduced it further to $0.1-0.2 \mathrm{nA}$ for $500-1000 \mathrm{~s}$. The precursor solutions we used were $0.5 \mathrm{M}$ aniline in $1.0 \mathrm{M} \mathrm{HCl}$ and $0.1 \mathrm{M}$ pyrrole in $0.1 \mathrm{M} \mathrm{LiClO}_{4}$. We monitored the growth of the CPNWs by using optical 

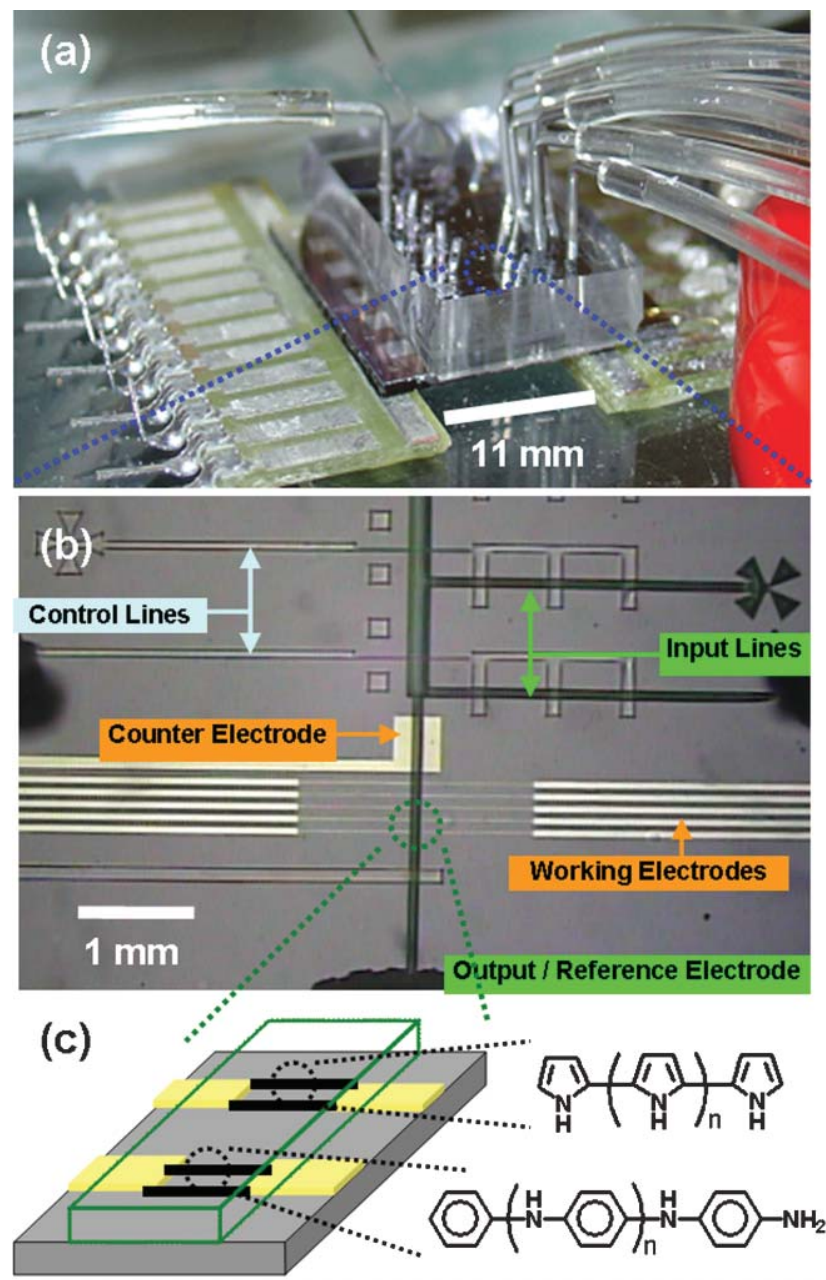

Fig. 1 (a) Actual view of the microfabricated and assembled integrated microfluidic device. (b) Optical micrograph of the integrated microfluidic device. Each microfluidic channel is $16 \mu \mathrm{m}$ high and $100 \mu \mathrm{m}$ wide. Each of the five pairs of electrode junctions is separated by a $2.0 \mu \mathrm{m}$-wide gap; the width and height of each electrode are $10 \mu \mathrm{m}$ and $50 \mathrm{~nm}$, respectively. (c) Schematic illustration of the electrochemical fabrication of CPNWs (in this case, polyaniline and polypyrrole) in the microfluidic channel.

microscopy (Zeiss, Axioskop2FS) and measured the conductance of the electrode junction periodically to assess the extent of polymerization.

We used scanning electron microscopy (SEM) to characterize the morphologies of the resulting polyaniline and polypyrrole nanowires (Fig. 2a and b, respectively) between the electrode junctions. The polyaniline nanowires, whose diameters ranged from 50 and $80 \mathrm{~nm}$, covered the entire exposed area of the working electrode and bridged the gap of the electrode junction. Polypyrrole nanowires were obtained with relatively larger diameters (ranging from 80 to $180 \mathrm{~nm}$ ) and better-defined morphologies. We performed these electropolymerizations in the absence of any flow, but it may be possible to control the diameter and uniformity of the CPNWs more precisely through careful manipulation of the flow rate and the concentrations of monomeric precursors. Nevertheless, compared with the morphology of polyaniline and polypyrrole electropolymerized in a bulk solution, ${ }^{6 b, c}$ the CPNWs which were grown in the microchannel
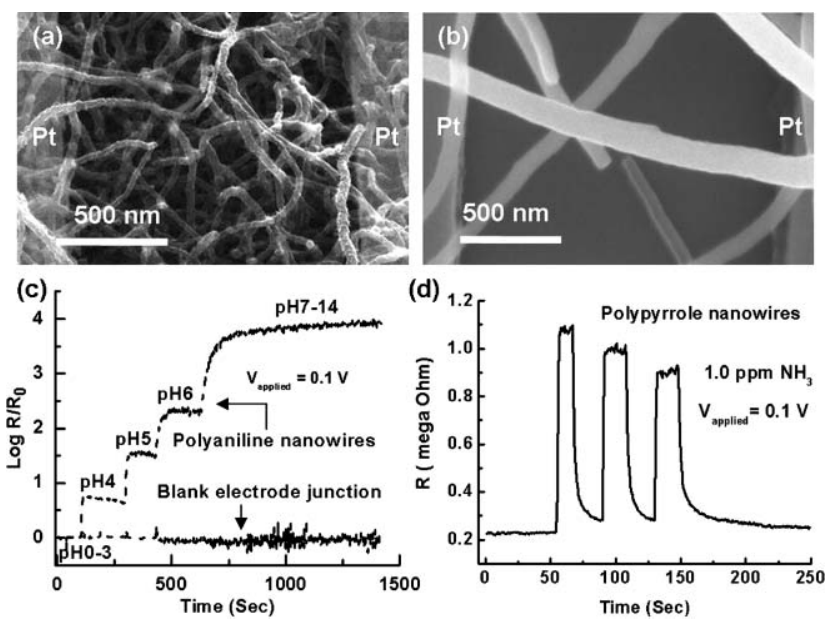

Fig. 2 SEM images of (a) polyaniline and (b) polypyrrole nanowires grown in the microfluidic channels. (c) Changes in the resistance of polyaniline nanowire electrode junction and a blank electrode junction upon varying the $\mathrm{pH}$ from 0 to 14 . (d) Reversible and reproducible response of a polypyrrole electrode junction to $\mathrm{NH}_{3}(1.0 \mathrm{ppm})$. The response intensities decayed gradually because the experiment was performed by repeatedly placing the array in an open container. For both of the sensing experiments, $0.1 \mathrm{~V}$ bias was applied.

are of better-defined morphologies and experimental fidelity. These CPNWs grew through a process of nucleation during the fast galvanostatic step and elongation during the subsequent steps. ${ }^{14}$ It is well established that carefully controlled nucleation of the monomer on the surface is critical for the growth of polymer nanostructures; fast nucleation and slow growth allow selfreorganization of CPNWs during the electrochemical polymerization. ${ }^{14}$ The turbulence-free environment ${ }^{13}$ in a microchannel may, therefore, encourage the formation of the well-defined and welloriented CPNWs. In addition, the electropolymerizations of aniline and pyrrole both take less than $40 \mathrm{~min}$ to produce the corresponding nanowires that bridge the electrode gap in the microfluidic device. In bulk solution, ${ }^{6 b, \mathrm{c}}$ however, these processes require almost $6 \mathrm{~h}$. Because of the nature of our method and the architecture of our device, it is apparent that the electrode array is individually addressable and can be further explored for CPNW sensors having different compositions and a range of functions.

The CPNWs electrode junctions can be used for sensing immediately after their fabrication within the microchannel. Fig. 2c presents the $\mathrm{pH}$ effect on the resistance of the polyaniline nanowires. In order to normalize the background conductance, we prepared 15 aqueous solutions (with $\mathrm{pH}$ ranging from 0 to 14) from a $1.0 \mathrm{M} \mathrm{NaCl}$ solution. The ionic strength of the solutions was adjusted to the same value with $\mathrm{NaCl}$, leading to the same background conductance for all measurements. We injected these solutions sequentially into the microchannel and recorded the change in resistance of the polyaniline nanowires using a Keithley 4200 analyzer. The resistance of the polyaniline nanowires was low between $\mathrm{pH} 0$ and 3; it increased logarithmically between 3 and 7 and above that the wires are non-conducting. Thus, at $\mathrm{pH}>7$ we just measured the solution conductance. This observation is consistent with the transition of the polymer from the conducting emeraldine salt form to the insulating emeraldine base form. ${ }^{15}$ These polyaniline nanowires responded quickly to the changing 
$\mathrm{pH}$ of the solution; equilibrium was reached within a few seconds because of the high surface-area-to-volume ratio of the nanowires and the rapid exchange of the solutions within the microfluidic chip. The time required for equilibration is significantly longer for nanowires grown in the bulk solution. ${ }^{6 \mathrm{~b}}$ The control curve in Fig. 2c was recorded using a blank electrode junction without CPNWs deposited. After removing the overlaying PDMS microfluidic component, the polypyrrole nanowires can be utilized for the real-time detection of $\mathrm{NH}_{3}$ gas. We demonstrated three cycles of the detection of $\mathrm{NH}_{3}(1.0 \mathrm{ppm})$. This experiment was performed by sequentially inserting and removing the polypyrrole electrode junction in and out of a $2.0 \mathrm{~L}$ plastic bottle containing $1.0 \mathrm{ppm} \mathrm{NH}_{3}$. The intensity of the response of the array toward $\mathrm{NH}_{3}$ decayed with each detection cycle because the experiment was conducted in an open system where the effective $\mathrm{NH}_{3}$ concentration was decreasing over time.

The method we describe in this paper opens up new possibilities in the fabrication of high-density, individually addressable CPNWs arrays for use in chemical and biological sensing. The integration of electropolymerization and microfluidic technology provides several important advantages that allow a simple and rapid fabrication of high-quality CPNW sensors and their immediate utilization in situ.

HRT thanks the UCLA SPORE Prostate Cancer and Cancer Research Coordinating Committee for the Developmental Grants. JRH and YLB thank the Institute for Collaborative Biotechnologies for a grant (DAAD19-030D-004).

\section{Notes and references}

$\dagger$ The electrode patterns (an array of five pairs of electrode junctions) employed for the electropolymerization of conducting polymer nanowires were fabricated by using standard photolithographic techniques. The device patterns were transferred from a photomask to a silicon (100) substrate covered with a $500 \mathrm{~nm}$-thick thermal oxide, followed by electron beam depositions of $5 \mathrm{~nm}$-thick $\mathrm{Ti}$ and $50 \mathrm{~nm}$-thick Pt layers. The spacing between the parallel electrodes was $c a .100 \mu \mathrm{m}$, and a $2.0 \mu \mathrm{m}$-wide gap existed between the tips of each pair of electrodes. Each electrode was linked internally with a millimeter-scale pad for connection with a potentiostat and semiconductor analyzer. Before integration with the microfluidic component, the electrodes were cleaned using piranha solution $\left(70 \%\right.$ conc. $\mathrm{H}_{2} \mathrm{SO}_{4}+30 \% \mathrm{H}_{2} \mathrm{O}_{2}$ ) for 2 min, rinsed with DI water, and then dried under a flow of $\mathrm{N}_{2}$. The integrated microfluidic components were fabricated using a soft lithography method. Two different molds were first fabricated by photolithographic processes for the production of the fluidic channels and control channels located in the respective layers of the microfluidic components. In this case, a channel height of $c a .16 \mu \mathrm{m}$ and a width of $100 \mu \mathrm{m}$ were chosen. PDMS polymer (RTV615A/B, GE) was used to fabricate the microfluidic components. Before fabricating the device, both the fluidic and control molds were exposed to trimethylsilyl chloride (TMSCl) vapor for 2-3 min. A well-mixed PDMS (GE, RTV 615 $\mathrm{A}$ and $\mathrm{B}, 5: 1)$ was spun onto the fluidic mold (1600 rpm, $60 \mathrm{~s}$, ramp $15 \mathrm{~s})$ to obtain the fluidic layer. Another portion of PDMS (GE, RTV 615 A and $\mathrm{B}, 30: 1)$ was poured onto the control mold located in a Petri dish to give a $5 \mathrm{~mm}$-thick control layer. The thin fluid layer and thick control layer were cured in an oven at $80{ }^{\circ} \mathrm{C}$ for $150 \mathrm{~min}$. After incubation, the thick control layer was peeled from the mold and holes were introduced onto the control layer for access to the pressure inlets. This layer was then trimmed, cleaned, and aligned onto the thin fluidic layer under a microscope. After baking at $80{ }^{\circ} \mathrm{C}$ for another $90 \mathrm{~min}$, the assembled layer was peeled from the control mold and the holes were punched to allow access to the solution. The aligned device was incubated in an oven at $80^{\circ} \mathrm{C}$ for $30 \mathrm{~min}$. The assembled layer was treated with oxygen plasma and placed on top of the electrode junctions on a silicon chip, and, finally, the device was incubated in an oven at $80{ }^{\circ} \mathrm{C}$ for $1 \mathrm{~h}$.

1 (a) F. Patolsky and C. M. Lieber, Mater. Today, 2005, 8, 20; (b) Z. Li, Y. Chen, X. Li, T. I. Kamins, K. Nauka and R. S. Williams, Nano Lett., 2004, 4, 245; (c) G. Zheng, F. Patolsky, Y. Cui, W. U. Wang and C. M. Lieber, Nat. Biotechnol., 2005, 23, 1294.

2 (a) J. Kong, N. R. Franklin, C. Zhou, M. G. Chapline, S. Peng, K. Cho and H. Dai, Science, 2000, 287, 622; (b) H. Dai, Acc. Chem. Res., 2002, 35, 1035; (c) A. Star, J.-C. P. Gabriel, K. Bradley and G. Gruner, Nano Lett., 2003, 3, 459; (d) A. Star, E. Tu, J. Niemann, J.-C. P. Gabriel, C. S. Joiner and C. Valcke, Proc. Natl. Acad. Sci. USA, 2006, 103, 921.

3 (a) J. Huang, S. Virji, B. H. Weiller and R. B. Kaner, J. Am. Chem. Soc., 2003, 125, 314; (b) B. J. S. Johnson, H. Wolf, J. A. S. Zalusky and M. A. Hillmyer, Chem. Mater., 2004, 16, 2909.

4 (a) C. Jerome and R. Jerome, Angew. Chem., Int. Ed., 1998, 37, 2488; (b) T. Hatano, A. H. Bae, M. Takeuchi, N. Fujita, K. Kaneko, H. Ihara, M. Takafuji and S. Shinkai, Angew. Chem., Int. Ed., 2004, 43, 465; (c) K. Ramanathan, M. A. Bangar, M. H. Yun, W. F. Chen, A. Mulchandani and N. V. Myung, Nano Lett., 2004, 4, 1237; (d) S. Park, J. H. Lim, S. W. Chung and C. A. Mirkin, Science, 2004, 303, 348; (e) Y. F. Ma, J. M. Zhang, G. J. Zhang and H. X. He, J. Am. Chem. Soc., 2004, 126, 7097; (f) V. Bocharova, A. Kiriy, H. Vinzelberg, I. Mönch and M. Stamm, Angew. Chem., Int. Ed., 2005, 44, 6391.

5 (a) D. H. Reneker and I. Chun, Nanotechnology, 1996, 7, 216; (b) D. H. Reneker, A. L. Yarin, H. Fong and S. Koombhongse, J. Appl. Phys., 2000, 87, 4531

6 (a) H. Liu, J. Kameoka, D. A. Czaplewski and H. G. Craighead, Nano Lett., 2004, 4, 671; (b) J. Wang, S. Chan, R. R. Carlson, Y. Luo, G. Ge, R. S. Ries, J. R. Heath and H. R. Tseng, Nano Lett., 2004, 4, 1693; (c) M. M. Alam, J. Wang, Y. Guo, S. P. Lee and H.-R. Tseng, J. Phys. Chem. B, 2005, 10, 12777; (d) E. S. Forzani, H. Zhang, L. A. Nagahara, I. Amlani, R. Tsui and N. Tao, Nano Lett., 2004, 4, 1785; (e) K. Ramanathan, M. A. Bangar, M. Yun, W. Chen, N. V. Myung and A. Mulchandani, J. Am. Chem. Soc., 2005, 127, 496.

7 (a) K. Jensen, Nature, 1998, 393, 735; (b) H. Wu, T. W. Odom, D. T. Chiu and G. M. Whitesides, J. Am. Chem. Soc., 2003, 125, 554; (c) A. Y. Fu, C. Spence, A. Scherer, F. H. Arnold and S. R. Quake, Nat. Biotechnol., 1999, 17, 1109; (d) M. A. Unger, H. P. Chou, T. Thorsen, A. Scherer and S. R. Quake, Science, 2000, 288, 113.

8 (a) J. Kobayashi, Y. Mori, K. Okamoto, R. Akiyama, M. Ueno, T. Kitamori and S. Kobayashi, Science, 2004, 304, 1305; (b) T. Kawaguchi, H. Miyata, K. Ataka, K. Mae and J. Yoshida, Angew. Chem., Int. Ed., 2005, 44, 2413; (c) C. C. Lee, G. Sui, A. Elizarov, C. J. Shu, Y.-S. Shin, A. N. Dooley, J. Huang, A. Daridon, P. Wyatt, D. Stout, H. C. Kolb, O. N. Witte, N. Satyamurthy, J. R. Heath,

M. E. Phelps, S. R. Quake and H.-R. Tseng, Science, 2005, 310, 1793.

9 B. H. Weigl and P. Yager, Science, 1999, 283, 346.

10 D. D. Cunningham, Anal. Chim. Acta, 2001, 429, 1.

11 S. A. Khan, A. Gunther, M. A. Schmidt and K. F. Jensen, Langmuir, 2004, 20, 8604.

12 A. Jahn, W. N. Vreeland, M. Gaitan and L. E. Locascio, J. Am. Chem. Soc., 2004, 126, 2674.

13 T. M. Squires and S. R. Quake, Rev. Mod. Phys., 2005, 77, 977.

14 L. Liang, J. Liu, C. F. Windisch, Jr., G. J. Exarhos and Y. Lin, Angew. Chem., Int. Ed., 2002, 41, 3665.

15 A. G. MacDiarmid, J. C. Chiang, A. F. Richter and A. J. Epstein, Synth. Met., 1987, 18, 285. 